\title{
Preface to the Special Issue on Thunderstorm Effects in the Atmosphere-Ionosphere System
}

\author{
F. J. Gordillo-Vázquez $\cdot$ A. Luque
}

Received: 27 September 2013/ Accepted: 30 September 2013/Published online: 18 October 2013

(C) Springer Science+Business Media Dordrecht 2013

\begin{abstract}
The first summer school of the "Thunderstorm Effects in the AtmosphereIonosphere System" (TEA-IS) funded by the European Science Foundation through its Research Network Programme took place in Torremolinos (Spain) on June 17-22, 2012. The meeting gathered almost 100 scientists with different backgrounds (plasma physics, electrical and signal engineering, geophysics, space physics and computational science) coming from 20 countries, both from inside and outside TEA-IS member countries. We very briefly comment here on the five review papers included in this Special Issue of Surveys in Geophysics devoted to the 2012 TEA-IS summer school.
\end{abstract}

This Special Issue is the outcome of the first summer school on "Thunderstorm Effects in the Atmosphere-Ionosphere System" (TEA-IS), held in Torremolinos (Spain) on June 17-22, 2012. TEA-IS is one of the Research Networking Programmes (RNP) in Life, Earth and Environmental Sciences currently funded by the European Science Foundation (ESF). It aims to foster the scientific collaboration between researchers from subscribing countries, and it has been operative since May 2011, and will end in April 2016. More than 100 scientists from 10 countries participate in the TEA-IS network; the currently subscribing countries are Denmark, Finland, France, Germany, Hungary, The Netherlands, Norway, Spain, Sweden and Switzerland.

The scientific focus of the TEA-IS network is the thunderstorm-coupling between different layers of the Earth's atmosphere. This is a broad topic that includes transient luminous events (TLEs) — such as sprites, halos, ELVES and jets-as well as terrestrial gamma-ray flashes (TGFs), the propagation of gravity waves and the electric field-driven microphysics of the atmosphere. Since the electrical activity inside thunderclouds is the main driver of these processes, the TEA-IS network also includes participants that

F. J. Gordillo-Vázquez $(\bowtie) \cdot A$. Luque Instituto de Astrofísica de Andalucía, IAA-CSIC, PO Box 3004, 18080 Granada, Spain e-mail: vazquez@iaa.es

A. Luque

e-mail: aluque@iaa.es 
specialize in the fundamentals of charging of thunderstorm particles, the physics of electric discharges and the applications and effects of lightning on weather.

Within the framework of the TEA-IS network, the Transient Plasmas in Planetary Atmospheres group at the Instituto de Astrofísica de Andalucía (IAA-CSIC) organized the 2012 Torremolinos summer school as a venue to gather researchers from all the above fields. Up to 96 participants, including 30 students, attended the summer school; they originated from 20 countries, both TEA-IS members and non-members. The program, the list of participants, the proceedings and some additional information are available in the web page of the summerschool http://www.teais.trappa.es/.

The present Special Issue compiles five review articles from speakers invited to the summer school. These reviews cover a broad range of the research topics in the TEA-IS network; other topics have been recently reviewed elsewhere, and we will refer the interested reader to the appropriate literature.

Being the origin of many atmosphere-ionosphere coupling processes, the lightning discharge plays a prominent role in the phenomena addressed in this Special Issue. Lightning physics is reviewed by Rakov (2013a) in the first article of our compilation. Focusing on negative cloud-to-ground (-CG) discharges, Rakov discusses the time evolution of a lightning discharge, including preliminary breakdown processes in the thundercloud, the propagation of lightning leaders and the connection of the channel to the ground. He also reviews briefly the effect of lightning on the mesosphere and lower ionosphere and the emission of hard radiation from lightning leaders.

The effects of a lightning discharge on the upper atmospheric layers are clearly affected by the characteristics of the discharge such as polarity, number of flashes, peak intensity and discharge duration. For a researcher interested in thunderstorm effects on the atmosphere-ionosphere system, it is therefore important to understand how these properties are obtained from remote sensing networks. This is the topic of our second article, also by Rakov (2013b), which deals with the radio emissions from lightning and with their detection in order to localize and remotely characterize the discharges. Particularly noteworthy is a short description of the most important lightning detection networks currently in operation.

The third article in the series, by Price (2013), describes how the Earth's climate ultimately drives the global lightning and thunderstorm activity. Conversely, lightning and thunderstorms can also both affect the climate in different ways, for instance, by producing large amounts of $\mathrm{NO}_{\mathrm{x}}$ (that generates tropospheric $\mathrm{O}_{3}$ ) and by transporting water vapor to the upper troposphere, respectively. Price (2013) also discusses the connection between severe weather phenomena (tornadoes, hailstorms, tropical storms, hurricanes or rainfall) and noticeable changes in key lightning features such as their number (more frequent) and polarity (increasing numbers of $+\mathrm{CG}$ discharges, even though there are still more $-\mathrm{CG}$ discharges that $+\mathrm{CG}$ ones), and on the importance of lightning observations as a valuable tool to monitor and predict severe weather scenarios. Finally, Price (2013) comments on how the aerosol loading in the atmosphere influences the occurrence of lightning.

A comprehensive overview of the key features of sprite streamers as derived from highspeed imaging observations is presented by Stenbaek-Nielsen et al. (2013) in the fourth article of this Special Issue. This paper goes through the different findings gained by the authors during recent years from several high-speed sprite imaging campaigns using equipment with frame rates of up to $32,000 \mathrm{fps}$, and with $30-50 \mathrm{~cm}$ focal length lenses reaching spatial resolutions down to $15 \mathrm{~m}$. Such observations have allowed StenbaekNielsen et al. (2013) to investigate the morphology and brightness of sprite streamers and to perform detailed analysis of the sprite streamer splitting dynamics from their high-speed 
imaging data. A rich discussion is also presented on the limits of applicability of streamer scaling laws when comparing some streamer features (velocity/diameter/brightness) in sprites and laboratory discharges under, respectively, varying and constant density conditions. Finally, the work by Stenbaek-Nielsen et al. (2013) poses several future challenges to be explored with next generation high-speed imaging systems. In particular, they point out five aspects worthy of future investigations, such as the double-headed features predicted by some models of streamers but not observed in high-frame-rate images, the relationship between velocity and width of sprite streamers, the general relationship between sprites and the driving lightning electric field produced by tropospheric thunderstorm activity, the observation of minimum size sprite streamers (that would require images with spatial resolutions below $10 \mathrm{~m}$ ) and further studies on the streamer splitting dynamics that would require the cameras' digital technology to go beyond their present limits.

The observations reviewed by Stenbaek-Nielsen et al. (2013) are taken into account for the development of sprite and halo models. These models are reviewed in the last paper of this Special Issue, by Pasko et al. (2013). Emphasizing results published after 2010, the central topic of that review is the emergence of sprite streamers and how they are distinct from the unstructured, diffuse emissions from a halo. Based on their recent work, they also offer insights into the so-called polarity asymmetry paradox of sprites: namely, why there are proportionally far fewer sprites generated by $-\mathrm{CG}$ discharges than by $+\mathrm{CG}$ ones, even after taking their different charge moment changes into account.

As we mentioned above, there are topics that have been left out of this Special Issue. High-energy processes such as TGFs and X-ray emissions from lightning are presently a very active research field. Recently, these processes were extensively reviewed by Dwyer et al. (2012) and we refer the reader to that work. Finally, a review article recently published by Füllekrug et al. (2013) in Surveys in Geophysics discusses in depth important scientific themes related to terrestrial gamma-ray flashes and relativistic electron beams above thunderclouds. In particular, the paper by Füllekrug et al. (2013) focuses on the detection of upward electromagnetic emissions from lightning discharges and energetic electron beams, and on how the Earth's atmosphere and near-Earth space are influenced by energetic charged particles striking the upper atmosphere.

Acknowledgements Both Guest Editors of this Special Issue acknowledge the European Science Foundation (ESF) for the support provided through the Research Network Programme (RNP) for the organization of the 2012 TEA-IS summer school in Torremolinos, Spain. They also thank Dr Torsten Neubert, of the Danish Space Research Center, for his leadership the TEA-IS Programme, and Prof Michael Rycroft, Editor of Surveys in Geophysics, for the invitation to prepare this Special Issue.

\section{References}

Dwyer JR, Smith DM, Cummer SA (2012) High-energy atmospheric physics: terrestrial gamma-ray flashes and related phenomena. Space Sci Rev 173:133. doi:10.1007/s11214-012-9894-0

Füllekrug M, Diver D, Pinçon JL, Phelps ADR, Bourdon A, Helling C, Blanc E, Honary F, Harrison RG, Sauvaud JA, Renard JB, Lester M, Rycroft M, Kosch M, Horne RB, Soula S, Gaffet S (2013) Energetic charged particles above thunderclouds. Surv Geophys 34:1. doi:10.1007/s10712-012-9205-Z

Pasko VP, Qin J, Celestin S (2013) Toward better understanding of sprite streamers: initiation, morphology, and polarity asymmetry. Surv Geophys. doi:10.1007/s10712-013-9246-y

Price CG (2013) Lightning applications in weather and climate research. Surv Geophys. doi:10.1007/ s10712-012-9218-7

Rakov VA (2013a) The physics of lightning. Surv Geophys. doi:10.1007/s10712-013-9230-6 
Rakov VA (2013b) Electromagnetic methods of lightning detection. Surv Geophys. doi:10.1007/s10712013-9251-1

Stenbaek-Nielsen HC, Kanmae T, McHarg MG, Haaland R (2013) High-speed observations of sprite streamers. Surv Geophys. doi:10.1007/s 10712-013-9224-4 